\title{
Circadian Rhythm Generation and Entrainment in Astrocytes
}

\author{
Laura M. Prolo, ${ }^{1}$ Joseph S. Takahashi, ${ }^{2}$ and Erik D. Herzog ${ }^{1}$ \\ ${ }^{1}$ Department of Biology, Washington University, St. Louis, Missouri 63130-4899, and ${ }^{2}$ Howard Hughes Medical Institute and Department of Neurobiology \\ and Physiology, Northwestern University, Evanston, Illinois 60208
}

\begin{abstract}
In mammals, the master circadian pacemaker is considered the suprachiasmatic nucleus (SCN) of the hypothalamus. The SCN consists of a heterogeneous population of neurons and relatively understudied glia. We investigated whether glia, like neurons, rhythmically express circadian genes. We generated pure cultures of cortical astrocytes from Period2::luciferase (Per2::luc) knock-in mice and Period1::luciferase (Per1::luc) transgenic rats and recorded bioluminescence as a real-time reporter of gene activity. We found that rat Per1::luc and mouse Per2::luc astroglia express circadian rhythms with a genetically determined period. These rhythms damped out after several days but were reinitiated by a variety of treatments, including a full volume exchange of the medium. If cultures were treated before damping out, the phase of Per1::luc rhythmicity was shifted, depending on the time of the pulse relative to the peak of Per1 expression. Glial rhythms entrained to daily $1.5^{\circ} \mathrm{C}$ temperature cycles and were significantly sustained when cocultured with explants of the adult SCN but not with cortical explants. Thus, multiple signals, including a diffusible factor(s) from the SCN, are sufficient to either entrain or restart circadian oscillations in cortical glia.
\end{abstract}

Key words: glia; suprachiasmatic nucleus; pacemaker; oscillator; Period1 gene; luciferase

\section{Introduction}

Most organisms have endogenous circadian clocks that coordinate physiological and behavioral rhythms and synchronize the organism to daily environmental cycles. The suprachiasmatic nucleus ( $\mathrm{SCN})$ of the hypothalamus is considered the master circadian pacemaker in mammals. The SCN is required for daily rhythms in behavior; lesions of the SCN abolish activity rhythms in mammals. Transplanting an explant of SCN into an SCNlesioned animal restores circadian rhythms with the donor's period (Ralph et al., 1990), and SCN neurons show sustained circadian gene expression, electrical activity, and neuropeptide secretion in the absence of external cues. Several other tissues, including the retina, olfactory bulb, and fibroblast cells, have been shown recently to fulfill the criteria of circadian oscillators: they have intrinsic circadian clocks with a genetically defined, temperature-compensated period that can be entrained to daily environmental cycles (Tosini and Menaker, 1996; Ruby et al., 1999; Herzog and Huckfeldt, 2003; Izumo et al., 2003; GranadosFuentes et al., 2004a,b). In some tissues, however, these rhythms do not seem to persist under constant conditions without input from the SCN (Balsalobre et al., 2000; Yamazaki et al., 2000; Pando et al., 2002). The SCN is thus believed to coordinate rhythms in the brain and body via a combination of diffusible

\footnotetext{
Received 0ct. 5, 2004; revised Nov. 14, 2004; accepted Nov. 19, 2004.

This work was supported by National Institutes of Health Grant MH63104. We are indebted to Dr. Hajime Tei (University of Tokyo, Tokyo, Japan) for the generous gift of the Per1::Iuc rats, to Dr. Martin Straume (University of Virginia, Charlottesville, VA) for analysis software, and to the Herzog laboratory for helpful comments.

Correspondence should be addressed to Erik Herzog, Box 1137, Department of Biology, Washington University, St. Louis, M0 63130-4899. E-mail: herzog@biology.wustl.edu.

DOI:10.1523/JNEUROSCI.4133-04.2005

Copyright $\odot 2005$ Society for Neuroscience $\quad 0270-6474 / 05 / 250404-05 \$ 15.00 / 0$
}

(Silver et al., 1996; Allen et al., 2001; Tousson and Meissl, 2004) and synaptic (Buijs and Kalsbeek, 2001) signals.

The SCN is composed of a heterogeneous population of cells, including astrocytes and multiple neuropeptidergic classes of neurons (Abrahamson and Moore, 2001). Although glial activity has been implicated in SCN function (van den Pol et al., 1992; Prosser et al., 1994; Shinohara et al., 2000), it is not known whether glia act as circadian pacemakers, modulate neural pacemaking, or are targets of SCN pacemaker neurons. We investigated whether astroglia are competent circadian oscillators able to respond to diffusible signals from the SCN.

\section{Materials and Methods}

Generation of primary astrocyte cultures. Wild-type and transgenic rats (Japanese Wistar; a generous gift from Dr. H. Tei, University of Tokyo, Tokyo, Japan) expressing $6.7 \mathrm{~kb}$ of the mouse Period1 (Per1) promoter driving firefly luciferase (Yamazaki et al., 2000) and knock-in mice (congenic on C57BL/6J) expressing an mPeriod2::luciferase (Per2::luc) fusion protein (Yoo et al., 2004) were maintained in a $12 \mathrm{~h}$ light/dark cycle in the Hilltop Animal Facility at Washington University. All of the procedures were approved by the Animal Care and Use Committee and conformed to National Institutes of Health guidelines.

Cortical glia cultures were generated following methods published previously (Noble and Mayer-Proschel, 1991). Briefly, neonates (postnatal days 2-6) were decapitated, and brains were placed in ice-cold HBSS supplemented with $100 \mathrm{U} / \mathrm{ml}$ penicillin, $100 \mu \mathrm{g} / \mathrm{ml}$ streptomycin (pen/ strep), $10 \mathrm{~mm}$ HEPES, and $4 \mathrm{~mm} \mathrm{NaHCO}_{3}$. Unless otherwise noted, all of the reagents were purchased from Sigma (St. Louis, MO). The meninges and blood vessels were dissected away. The cerebral hemispheres were then minced with scissors and incubated in 1\% DNaseI, $0.005 \%$ trypsin, and $0.002 \%$ EDTA (Invitrogen, Carlsbad, CA) at $37^{\circ} \mathrm{C}$ for $15 \mathrm{~min}$. The supernatant was forced through a sterile nylon mesh into $3 \mathrm{ml}$ of horse serum (Invitrogen), centrifuged at $1000 \mathrm{rpm}$ for $5 \mathrm{~min}$, and resuspended 
in Minimum Essential Medium (MEM) (Invitrogen) supplemented with $10 \%$ horse serum, pen/strep, and $33 \mathrm{~mm}$ glucose. The cells were plated in a $75 \mathrm{~cm}^{2}$ flask and incubated at $37^{\circ} \mathrm{C}$ with $5 \% \mathrm{CO}_{2}$ and $95 \%$ air.

Cultures were passaged after reaching confluency $\sim 10 \mathrm{~d}$ after plating. The flasks were banged and then rinsed with HBSS to remove microglia. The remaining cells were then incubated in $0.05 \%$ trypsin and $0.02 \%$ EDTA at $37^{\circ} \mathrm{C}$ for $10 \mathrm{~min}$. The flasks were again banged to detach the astrocytes, which were centrifuged at $1000 \mathrm{rpm}$ for $5 \mathrm{~min}$, resuspended in MEM with $10 \%$ horse serum, and then plated in a flask or onto a coverslip. Data were collected from cultures that had been passaged between one and seven times. We counted cell density using a hemacytometer. All of the astrocyte cultures for bioluminescence and immunocytochemistry assays were made using identical methods and plated at 1300 cells $/ \mathrm{mm}^{2}$ on poly-D-lysine-coated coverslips in a $10 \mathrm{~mm}$ Sylgard (Dow Corning, Midland, MI) gasket. Some cultures were fixed and immunolabeled at various times during bioluminescence recording to confirm that cell density and glial purity did not change.

Immunocytochemistry. Immunolabeling was performed on Per1::luc cortical glia and wild-type SCN dispersals (Herzog et al., 1998) after 8-10 d in vitro. Cells were fixed in $4 \%$ paraformaldehyde and $4 \%$ sucrose for $15 \mathrm{~min}$, rinsed in $0.1 \mathrm{M}$ PBS, permeablized in $0.25 \%$ Triton X-100/PBS for $5 \mathrm{~min}$, rinsed in PBS, and then blocked in 10\% bovine serum albumin (BSA) for 30 min at $37^{\circ} \mathrm{C}$. Glial cultures and $\mathrm{SCN}$ dispersals were incubated for $2 \mathrm{~h}$ at $37^{\circ} \mathrm{C}$ with primary antibodies against glial fibrillary acidic protein (GFAP) (rabbit anti-GFAP, 1:100 in 3\% BSA/PBS, $n=8$; Sigma), neurofilament 2 (NF2) (rabbit anti-NF2, 1:1000, $n=8$ glial and 3 SCN cultures; Sigma), or microtubule-associated protein 2 (MAP2) (mouse anti-MAP2, 1:2000, $n=3$ glial and 4 SCN cultures; Calbiochem, La Jolla, CA), rinsed in PBS, and incubated with an appropriate secondary antibody for $1 \mathrm{~h}$ at $37^{\circ} \mathrm{C}$ in the dark (GFAP, Cy3 goat anti-rabbit, 1:100; NF2, FITC donkey anti-rabbit, 1:200; MAP2, Texas Red donkey anti-mouse, 1:200; Jackson ImmunoResearch, West Grove, PA). Glial cultures were counterstained for 2 min with $100 \mathrm{~nm} 4^{\prime}, 6^{\prime}$ diamidino-2-phenylindole dihydrochloride (DAPI) (Molecular Probes, Eugene, OR). The coverslips were mounted in $50 \%$ glycerol/PBS, and fluorescence was imaged with a digital camera (Retiga-EX; Qimaging, Burnaby, British Columbia, Canada) at 20× magnification (TE200; Nikon, Melville, NY). We determined cell density from DAPI-labeled nuclei in five randomly selected fields $(260 \times 260 \mu \mathrm{m})$ per coverslip (Northern Eclipse software; Empix Imaging, North Tonawanda, NY).

Bioluminescence recording. Per::luc activity was continuously monitored (HC135-11MOD photomultiplier tube; Hamamatsu, Shizouka, Japan) from cortical glia plated on a glass coverslip in a sealed Petri dish with $1 \mathrm{ml}$ of serum-free DMEM supplemented with 2\% B27 (Invitrogen), pen/strep, 10 mM HEPES, $0.35 \mathrm{~g} / \mathrm{L} \mathrm{NaHCO}_{3}, 4.5 \mathrm{~g} / \mathrm{L}$ D-glucose, $2 \%$ L-glutamine, and 0.1 mu beetle luciferin (Promega, Madison, WI) as described previously (Abe et al., 2002). The medium was changed only in the phase-shift experiments when the full volume was exchanged at a defined time after plating.

For coculture experiments, a tissue explant (coronal, $300 \mu \mathrm{m}$ thick) of either the SCN or somatosensory or motor cortex was grown on a Millicell-CM membrane (Millipore, Bedford, MA) $500 \mu \mathrm{m}$ above a coverslip of Per1::luc cortical astrocytes. Surgery for the explants was performed $2 \mathrm{~h}$ before lights off in the animal colony.

Data analysis. The number of cycles, the amplitude, and the period of Per::luc expression in glial cultures was determined using methods described previously (Abe et al., 2002). The time of peak expression from each cycle defined the phase and period of each culture. A culture was considered no longer rhythmic when the peak-to-trough amplitude of bioluminescence was $<10 \%$ of the peak-to-trough height on the second circadian cycle or when the period was outside $18-30 \mathrm{~h}$. We chose these criteria because they tended to be fulfilled on the same circadian cycle and because an independent measure of period (fast Fourier transform-nonlinear least squares) (Herzog et al., 1998) usually found no significant circadian rhythmicity when the amplitude dropped to this level.

\section{Results}

\section{Pure glial cultures express damped circadian rhythms in} Per1::luc and Per2::luc activity

We established primary cultures of cortical astroglia derived from Per1::luc transgenic rats and Per2::luc knock-in mice. All of the

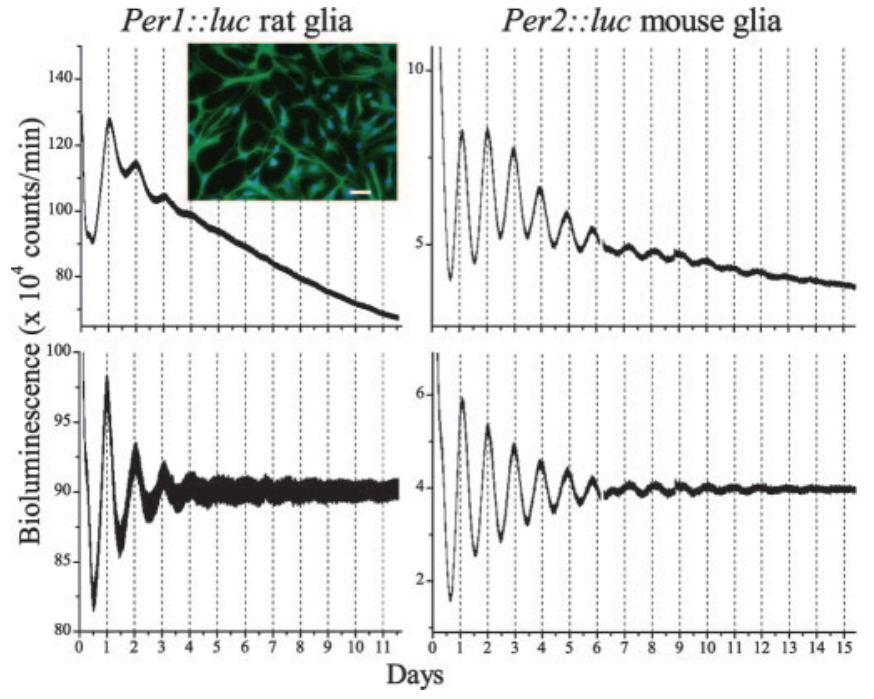

Figure 1. Glia express circadian periodicity independent of interactions with neurons. Inset, All cells stained for GFAP (green), indicating that the culture contains only astrocytes. Cells were counterstained with the nuclear dye DAPI (blue). Cortical glia $(100,000)$ were plated at 1300 cells $/ \mathrm{mm}^{2}$. Scale bar, $40 \mu \mathrm{m}$. Representative cultures of pure rat (Per1::/uc, top left) and mouse (Per2::Iuc glia, top right) show circadian rhythms and a gradual decrease in the overall bioluminescence level with time since plating. Detrended data from the same cultures (bottom) illustrate that the peak-to-trough amplitude of bioluminescence decreases with time in vitro.

cells in these cultures immunolabeled for GFAP, a specific marker for astrocytes (Fig. 1, inset). No cells labeled for two neuron-specific markers (MAP2 or NF2; see Materials and Methods). These cultures expressed circadian rhythms in bioluminescence, which damped out within 1 week after the cells were plated (Fig. 1). Rhythmicity did not depend on passage number. Interestingly, the average period of Per1::luc $(24.5 \pm 0.5 \mathrm{~h}$; mean \pm SEM; $n=14$ ) was significantly longer than that of Per2::luc (22.4 $\pm 0.1 \mathrm{~h} ; n=8 ; p=0.006$; Student's $t$ test).

To determine whether the exponential decline in rhythm amplitude was a consequence of compromised health, we assessed the response of damped cultures to a variety of treatments. Rhythms could be reinitiated by a $10 \mathrm{~min}$ exposure to calcimycin $(1 \mu \mathrm{g} / \mathrm{ml} ; n=2$ of 2$)$ or forskolin $(10 \mu \mathrm{M} ; n=8$ of 8$)$. Although exposure at $22^{\circ} \mathrm{C}$ for $10 \mathrm{~min}$ was not sufficient to restart rhythms ( $n=4$ of 4 ), a full exchange of the culture medium reliably resulted in resumed rhythmicity with an amplitude similar to that seen on the second cycle after plating $(n=30$ of 30$)$.

\section{Rhythms in Per1::luc glia can be reset depending on circadian phase}

In addition to the ability to generate daily rhythms, circadian pacemakers must be able to reset their oscillations to local time. To determine whether glia can be phase shifted, we measured the response of oscillating and damped glial cultures to a medium change given at a defined time after plating. We exchanged the medium during either the $24-48 \mathrm{~h}$ after plating for oscillating cultures or the 11th day after plating for damped cultures ( $n=3$ cultures for time points $0-20 ; n=2$ for $t=24)$ (Fig. 2). All of the cultures showed a rapid induction of Per1-driven bioluminescence and subsequent rhythmicity. Oscillating glia peaked at times that depended on the time of the treatment. For example, those cultures that received their medium change $24 \mathrm{~h}$ after plating peaked $27.6 \pm 0.3 \mathrm{~h}$ later, but those treated $36 \mathrm{~h}$ after plating peaked $33.0 \pm 1.0 \mathrm{~h}$ later or $5.4 \mathrm{~h}$ after those pulsed at $24 \mathrm{~h}$ after plating. By defining the time when the first cultures were treated 
(24 h after plating for oscillating cultures or $280 \mathrm{~h}$ after plating for damped cultures) as time 0 , we constructed a phase-response curve (PRC) with this 5.4 h delay occurring at time 12 ( $36 \mathrm{~h}$ after plating). Treatments at previous times produced smaller phase delays, and treatments at later times produced phase advances. In contrast, the PRC of glia pulsed when they no longer expressed Per1::luc rhythms was high amplitude, always resetting the subsequent rhythm to peak $\sim 28.1 \pm 0.3 \mathrm{~h}$ $(n=20)$ after the time of the pulse. Thus, when Per1::luc expression was oscillating, the induced phase shifts were smaller and phase dependent (i.e., a "type 1" PRC), and, when Per1::luc was constitutively low, rhythms resumed at the same phase regardless of the time of treatment (i.e., a “type 0" PRC).

\section{Temperature cycles entrain Per1::luc rhythms in glia}

To assess whether rhythms in glia can be entrained to physiologically relevant temperature changes, we exposed glial cultures to five $1.5^{\circ} \mathrm{C}$ cycles. We compared bioluminescence rhythms in cultures $(n=$ 9) exposed at $36.1^{\circ} \mathrm{C}$ with those exposed to a daily cycle of $12 \mathrm{~h}$ at $36.8^{\circ} \mathrm{C}$ and $12 \mathrm{~h}$ at $35.3^{\circ} \mathrm{C}(n=10)$. Glia responded to the initial drop in temperature by rapidly increasing Per $1::$ luc expression, peaking $\sim 11$

$\mathrm{h}$ after their last peak at $36.8^{\circ} \mathrm{C}$ (Fig. 3 ). On successive days, expression was synchronized across all of the cultures, peaking progressively closer to the cool-to-warm transition. After the last temperature cycle, Per $1:: l u c$ activity peaked $20.0 \pm 0.2 \mathrm{~h}$ after the last cool-to-warm transition and continued to oscillate from this phase for 7 more days when cultures at $36.1^{\circ} \mathrm{C}$ had previously damped out. These results indicate that the temperature cycle entrained and sustained rhythmicity in glia that would have otherwise damped out.

\section{The adult SCN modestly sustains Per1::luc rhythms in cocultured glia}

To address whether other physiologically relevant signals might also suffice to entrain and sustain periodicity in glial cultures, we measured bioluminescence from Perl::luc glia cocultured with a wild-type SCN explant placed on a membrane $0.5 \mathrm{~mm}$ above the glia (Fig. 4). As in cultures of pure glia, the amplitude of Per1::luc rhythmicity decreased over the first $5 \mathrm{~d}$ after plating. However, $30 \%$ of glial cultures exposed to an adult SCN explant $(n=6$ of 20 ) and $17 \%$ of those with a neonatal SCN explant ( $n=2$ of 12 ) expressed circadian rhythms for $7 \mathrm{~d}$ or more after plating when no cultures of pure glia ( 0 of 19$)$ were significantly rhythmic. The amplitude of rhythmicity was significantly greater in glial cultures cocultured with adult SCN than that of glia alone on the seventh and eighth circadian cycles (cycle 7, $F_{(4,63)}=4.6, p=$ 0.002 ; cycle $8, F_{(4,87)}=5.2, p<0.001$; one-way ANOVA with Tukey's post hoc test). In contrast, cocultures of neonatal SCN, adult cortex $(n=5)$, or neonatal cortex $(n=12)$ did not significantly sustain the amplitude of Per $1:$ :luc rhythms in glia. After $8 \mathrm{~d}$ of recording, the average number of cycles expressed by glia was
$4.4 \pm 0.2$ when cultured alone, $6.4 \pm 0.4$ when cultured with an adult SCN, $5.0 \pm 0.5$ when cultured with a neonatal SCN, $5.4 \pm$ 0.2 when cultured with an adult cortical explant, and $3.5 \pm 0.4$ when cultured with a neonatal cortical explant. Thus, only the adult SCN explant was able to significantly sustain glial rhythms under these conditions $\left(F_{(4,63)}=8.4, p<0.0001\right.$; one-way ANOVA with Tukey's post hoc test).

\section{Discussion}

Although Per1 and Per2 expression have been reported in a wide variety of neural and non-neural cell types (Balsalobre et al., 1998, 2000; Yamazaki et al., 2000; Stokkan et al., 2001; Abe et al., 2002; Yoo et al., 2004), this is the first demonstration that astrocytes express clock genes and can function as damped circadian oscillators independent of interactions with neurons. Cultured astrocytes express Per1::luc and Per2::luc rhythmically with a period close to $24 \mathrm{~h}$. Although one report specifically noted the lack of Per1::luc expression in glia (Yamaguchi et al., 2003), it may be that Per1 levels in astrocytes are substantially lower or more diffuse than in neurons, making it less visible in imaging experiments of the SCN slice. Bioluminescence of individual astrocytes can be imaged in our cultures using a deeply cooled digital camera (data not shown). Furthermore, our in vitro findings are consistent with the in vivo rhythms in GFAP immunoreactivity (Lavialle and Serviere, 1993) and light induction of c-Fos in glia of the SCN (Bennett and Schwartz, 1994). Similar to other circadian oscillators, glia are able to be phase shifted and entrain to daily time cues. In addition, the periods in murine and rat glia were significantly different, paralleling period differences seen in locomotor behavior of the two species. Thus, the circadian period of 

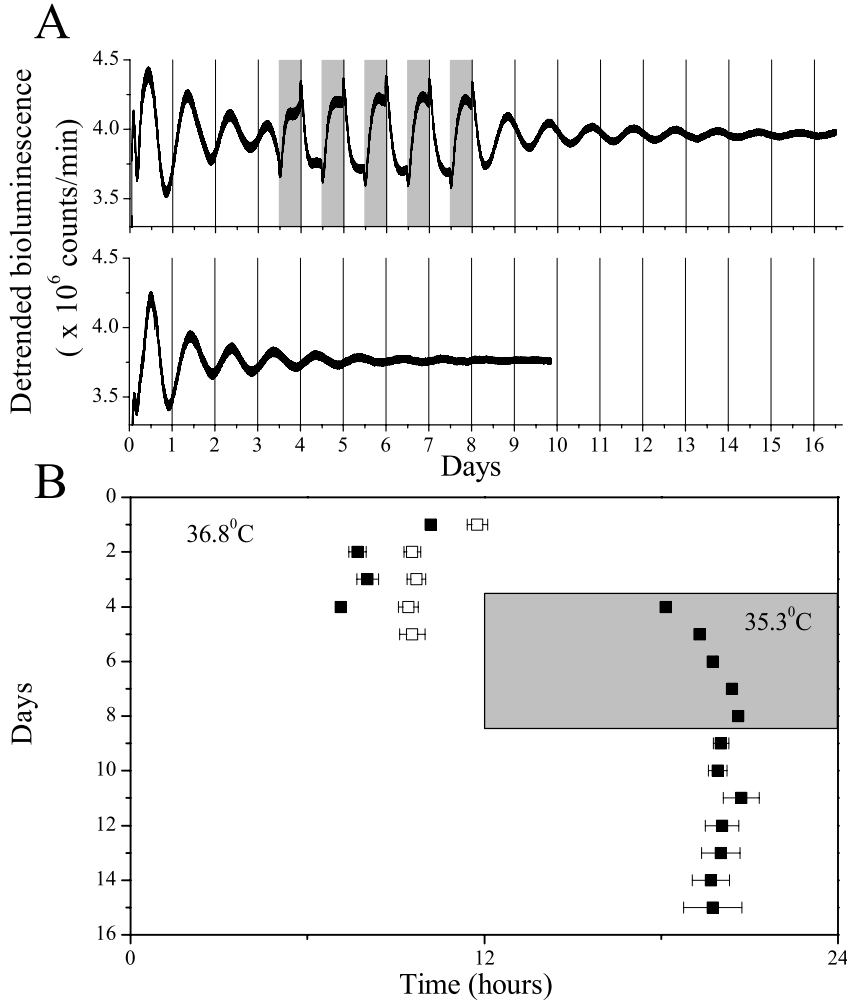

Figure 3. Glia entrain to a temperature cycle. $A$, Representative bioluminescence recordings from glia cultured in a $1.5^{\circ} \mathrm{C}$ temperature cycle (top) or at constant temperature (bottom). Glia responded to a daily decrease in temperature (gray rectangles) by increasing levels of Per 1::/uc expression. Eight days after plating, glia that experienced the temperature changes continued to oscillate, whereas glia that did not experience the temperature cycles damped. $B$, Times of peak expression for glia that experienced either cyclic (filled squares) or constant (open squares) temperature. Cooling to $35.3^{\circ} \mathrm{C}$ (gray rectangle) on day 4 after plating caused a peak in Per1::I/uc expression $11 \mathrm{~h}$ after the previous phase, $6 \mathrm{~h}$ after the warm-to-cool transition. By the fourth day of the temperature cycle, Per1::Iuc reliably peaked $\sim 8 \mathrm{~h}$ after the warm-to-cool transition. After returning to constant temperature, glia free ran from this new phase. Error bars represent SEM.

glia seems to be genetically determined, as has been shown for the SCN (Liu et al., 1997; Herzog et al., 1998; Albus et al., 2002; Nakamura et al., 2002), retina (Tosini and Menaker, 1996), and fibroblasts (Yagita et al., 2001). It will be important to address the role of glia as modulators and targets of neuronal rhythms.

Unlike the sustained oscillations seen in neurons from the SCN (Welsh et al., 1995), rhythms in cortical glia are undetectable within 1 week in vitro. Damping may thus reflect an essential difference in molecular rhythm generation between neurons and cortical glia. The exponential decrease in rhythm amplitude is not related to a loss of cells, a general decline in the health of the cells, or the density at which they are plated. Instead, damping may reflect gradual desynchrony among a population of sustained oscillators or a loss of rhythmicity in individual cells (Robertson and Takahashi, 1988). The fact that the PRC of oscillating glial cultures differs from that of arrhythmic cultures suggests a fundamental change in the amplitudes of individual oscillators, but future experiments measuring rhythms in individual cells are needed.

Importantly, Per $1::$ luc rhythms in glia can be phase shifted and entrained to a physiologically relevant cycle, suggesting that, in vivo, glia continue to oscillate as a result of periodic signaling. We found that forskolin, calcimycin, and medium changes were all sufficient to reinitiate rhythms in glia. These stimuli may all be acting through a common mechanism on the clock, such as the
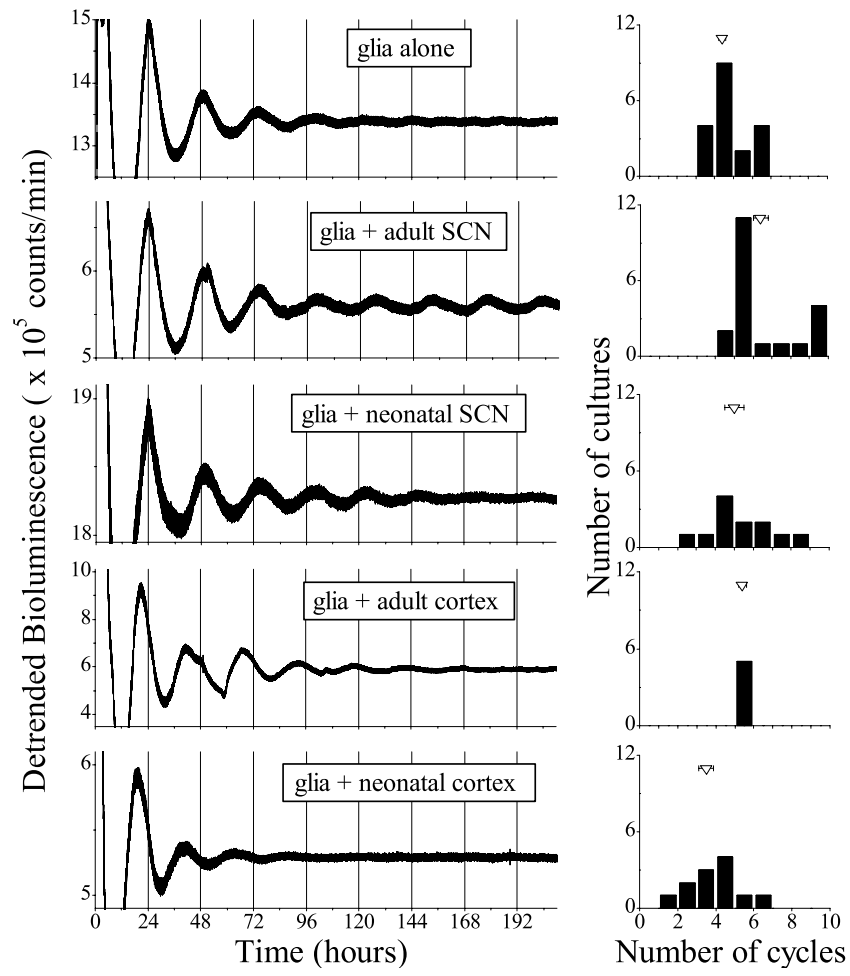

Figure 4. The adult SCN appears to sustain circadian rhythms in glia. A, Representative Per1::Iuc bioluminescence recording from glia plated alone or cocultured with an explant of SCN or cortex from an adult or neonatal rat. $B$, The distribution of the number of circadian cycles expressed by glial cultures for each of the conditions presented in $A$. The mean duration of rhythmicity after $10 \mathrm{~d}$ of recording (triangles) was increased when glia were cocultured with an adult SCN. Error bars represent SEM.

elevation of intracellular $\mathrm{Ca}^{2+}$ levels. Temperature changes are sufficient to entrain glial cultures but are unlikely to be required because manipulations such as exposure to SCN explants sustained glial rhythms without changing temperature. The ability to work with a homogeneous population of rhythmic primary cells will greatly facilitate future studies of entrainment mechanisms.

We found that the adult SCN is better able to sustain Per $1:: l u c$ rhythms in glia than an explant of neonatal SCN. This communication is likely via a diffusible signal, because the tissue was grown on a membrane $500 \mu \mathrm{m}$ above the glia. Because adult SCN was more effective on glia than neonatal SCN, this signal may not be produced in abundance during the first week after birth. Because glial rhythms were not significantly sustained when cocultured with an explant of cortex, the signal may be specific to the SCN. These results are consistent with previous studies that provide evidence for the SCN communicating via humoral signals (Silver et al., 1996; Tousson and Meissl, 2004). Importantly, glia express receptors for candidate diffusible factors such as arginine vasopressin, vasoactive intestinal polypeptide, GABA, and nitric oxide (Hosli and Hosli, 1993; Baltrons and Garcia, 2001). To assess whether the signal from the SCN to glia is periodic or tonic in its action, it will be important to test whether the SCN from a circadian mutant or an animal reared in a reverse light schedule is able to change period or phase in glial cultures.

Together, these results indicate that astrocytes are competent circadian oscillators, expressing rhythms that can be phase shifted and entrained by physiologically relevant signals. Future work should determine whether glia are involved with rhythm generation or coordination or are target cells of SCN neurons in vivo. 


\section{References}

Abe M, Herzog ED, Yamazaki S, Straume M, Tei H, Sakaki Y, Menaker M, Block GD (2002) Circadian rhythms in isolated brain regions. J Neurosci 22:350-356.

Abrahamson EE, Moore RY (2001) Suprachiasmatic nucleus in the mouse: retinal innervation, intrinsic organization and efferent projections. Brain Res 916:172-191.

Albus H, Bonnefont X, Chaves I, Yasui A, Doczy J, van der Horst GT, Meijer JH (2002) Cryptochrome-deficient mice lack circadian electrical activity in the suprachiasmatic nuclei. Curr Biol 12:1130-1133.

Allen G, Rappe J, Earnest DJ, Cassone VM (2001) Oscillating on borrowed time: diffusible signals from immortalized suprachiasmatic nucleus cells regulate circadian rhythmicity in cultured fibroblasts. J Neurosci 21:7937-7943.

Balsalobre A, Damiola F, Schibler U (1998) A serum shock induces circadian gene expression in mammalian tissue culture cells. Cell 93:929-937.

Balsalobre A, Marcacci L, Schibler U (2000) Multiple signaling pathways elicit circadian gene expression in cultured Rat-1 fibroblasts. Curr Biol 10:1291-1294.

Baltrons MA, Garcia A (2001) The nitric oxide/cyclic GMP system in astroglial cells. Prog Brain Res 132:325-337.

Bennett MR, Schwartz WJ (1994) Are glia among the cells that express immunoreactive c-Fos in the suprachiasmatic nucleus? NeuroReport 5:1737-1740.

Buijs RM, Kalsbeek A (2001) Hypothalamic integration of central and peripheral clocks. Nat Rev Neurosci 2:521-526.

Granados-Fuentes D, Prolo LM, Abraham U, Herzog ED (2004a) The suprachiasmatic nucleus entrains, but does not sustain, circadian rhythmicity in the olfactory bulb. J Neurosci 24:615-619.

Granados-Fuentes D, Saxena MT, Prolo LM, Aton SJ, Herzog ED (2004b) Olfactory bulb neurons express functional, entrainable circadian rhythms. Eur J Neurosci 19:898-906.

Herzog ED, Huckfeldt RM (2003) Circadian entrainment to temperature, but not light, in the isolated suprachiasmatic nucleus. J Neurophysiol 90:763-770.

Herzog ED, Takahashi JS, Block GD (1998) Clock controls circadian period in isolated suprachiasmatic nucleus neurons. Nat Neurosci 1:708-713.

Hosli E, Hosli L (1993) Receptors for neurotransmitters on astrocytes in the mammalian central nervous system. Prog Neurobiol 40:477-506.

Izumo M, Johnson CH, Yamazaki S (2003) Circadian gene expression in mammalian fibroblasts revealed by real-time luminescence reporting: temperature compensation and damping. Proc Natl Acad Sci USA 100:16089-16094.

Lavialle M, Serviere J (1993) Circadian fluctuations in GFAP distribution in the Syrian hamster suprachiasmatic nucleus. NeuroReport 4:1243-1246.

Liu C, Weaver DR, Strogatz SH, Reppert SM (1997) Cellular construction of a circadian clock: period determination in the suprachiasmatic nuclei. Cell 91:855-860.

Nakamura W, Honma S, Shirakawa T, Honma K-I (2002) Clock mutation lengthens the circadian period without damping rhythms in individual SCN neurons. Nat Neurosci 5:399-400.

Noble M, Mayer-Proschel M (1991) Culture of astrocytes, oligodendrocytes and O-2A progenitor cells. In: Culturing nerve cells (Banker G, Goslin K, eds), pp 499-531. Cambridge, MA: MIT.

Pando MP, Morse D, Cermakian N, Sassone-Corsi P (2002) Phenotypic rescue of a peripheral clock genetic defect via SCN hierarchical dominance. Cell 110:107-117.

Prosser RA, Edgar DM, Heller HC, Miller JD (1994) A possible glial role in the mammalian circadian clock. Brain Res 643:296-301.

Ralph MR, Foster RG, Davis FC, Menaker M (1990) Transplanted suprachiasmatic nucleus determines circadian period. Science 247:975-978.

Robertson LM, Takahashi JS (1988) Circadian clock in cell culture. II. In vitro photic entrainment of melatonin oscillation from dissociated chick pineal cells. J Neurosci 8:22-30.

Ruby NF, Burns DE, Heller HC (1999) Circadian rhythms in the suprachiasmatic nucleus are temperature-compensated and phase-shifted by heat pulses in vitro. J Neurosci 19:8630-8636.

Shinohara K, Honma S, Katsuno Y, Honma K-I (2000) Circadian release of excitatory amino acids in the suprachiasmatic nucleus culture is $\mathrm{Ca}^{(2+)}$ independent. Neurosci Res 36:245-250.

Silver R, LeSauter J, Tresco PA, Lehman MN (1996) A diffusible coupling signal from the transplanted suprachiasmatic nucleus controlling circadian locomotor rhythms. Nature 382:810-813.

Stokkan KA, Yamazaki S, Tei H, Sakaki Y, Menaker M (2001) Entrainment of the circadian clock in the liver by feeding. Science 291:490-493.

Tosini G, Menaker M (1996) Circadian rhythms in cultured mammalian retina. Science 272:419-421.

Tousson E, Meissl H (2004) Suprachiasmatic nuclei grafts restore the circadian rhythm in the paraventricular nucleus of the hypothalamus. J Neurosci 24:2983-2988.

van den Pol AN, Finkbeiner SM, Cornell-Bell AH (1992) Calcium excitability and oscillations in suprachiasmatic nucleus neurons and glia in vitro. J Neurosci 12:2648-2664.

Welsh DK, Logothetis DE, Meister M, Reppert SM (1995) Individual neurons dissociated from rat suprachiasmatic nucleus express independently phased circadian firing rhythms. Neuron 14:697-706.

Yagita K, Tamanini F, Der Horst GT, Okamura H (2001) Molecular mechanisms of the biological clock in cultured fibroblasts. Science 292:278-281.

Yamaguchi S, Isejima H, Matsuo T, Okura R, Yagita K, Kobayashi M, Okamura H (2003) Synchronization of cellular clocks in the suprachiasmatic nucleus. Science 302:1408-1412.

Yamazaki S, Numano R, Abe M, Hida A, Takahashi R, Ueda M, Block GD, Sakaki Y, Menaker M, Tei H (2000) Resetting central and peripheral circadian oscillators in transgenic rats. Science 288:682-685.

Yoo S-H, Yamazaki S, Lowrey PL, Shimomura K, Ko CH, Buhr ED, Siepka SM, Hong H-K, Oh WJ, Yoo OJ, Menaker M, Takahashi JS (2004) Period2::luciferase real-time reporting of circadian dynamics reveals persistent circadian oscillations in mouse peripheral tissues. Proc Natl Acad Sci USA 12:1-8. 